\title{
Reflex-mediated dynamic neuromuscular stabilization in stroke patients: EMG processing and ultrasound imaging
}

\author{
Hyun S. Yoon ${ }^{\mathrm{a}, \mathrm{b}}$ and Joshua (Sung) H. You ${ }^{\mathrm{b}, *}$ \\ ${ }^{\text {a }}$ Chungnam National University Hospital, Daejeon, Korea \\ ${ }^{\mathrm{b}}$ Sports $\cdot$ Movement Institue \& Technology, Department of Physical Therapy Program, Yonsei \\ University, Wonju, Kangwon-do, Korea
}

\begin{abstract}
.
BACKGROUND: Postural core instability is associated with poor dynamic balance and a high risk of serious falls. Both neurodevelopmental treatment (NDT) and dynamic neuromuscular stabilization (DNS) core stabilization exercises have been used to improve core stability, but the outcomes of these treatments remain unclear.

OBJECTIVE: This study was undertaken to examine the therapeutic effects of NDT and DNS core stabilization exercises on muscular activity, core stability, and core muscle thickness.

METHODS: Ten participants (5 healthy adults; 5 hemiparetic stroke patients) were recruited. Surface electromyography (EMG) was used to determine core muscle activity of the transversus abdominis/internal oblique (TrA/IO), external oblique (EO), and rectus abdominis (RA) muscles. Ultrasound imaging was used to measure transversus abdominals/internal oblique (TrA/IO) thickness, and a pressure biofeedback unit (PBU) was used to measure core stability during the DNS and NDT core exercise conditions. Data are reported as median and range and were compared using nonparametric Mann - Whitney U test and Wilcoxon signed rank test at $p<0.05$.

RESULTS: Both healthy and hemiparetic stroke groups showed greater median EMG amplitude in the TrA/IO muscles, core stability, and muscle thickness values during the DNS exercise condition than during the NDT core exercise condition, respectively $(p<0.05)$. However, the relative changes in the EMG amplitude, core stability, and muscle thickness values were greater during the DNS exercise condition than during the NDT core exercise condition in the hemiparetic stroke patient group ( $p<$ $0.05)$.

CONCLUSIONS: Our novel results provide the first clinical evidence that DNS is more effective than NDT in both healthy and hemiparetic stroke subjects to provide superior deep core muscle activation, core stabilization, and muscle thickness. Moreover, such advantageous therapeutic benefits of the DNS core stabilization exercise over the NDT exercise were more apparent in the hemiparetis stroke patients than normal controls.
\end{abstract}

Keywords: Electromyography signal processing, ultrasound imaging, dynamic neuromuscular stabilization

\section{Introduction}

Postural core instability is associated with poor dynamic balance and a high risk of serious falls [1]. Neurodevelopmental treatment (NDT) is commonly used to improve postural core stability, but the outcomes of this treatment remain unclear. NDT encourages 'conscious' and active postural core stabilization to upright the spine and involves activating abdominal muscles while maintaining the neutral

\footnotetext{
${ }^{*}$ Corresponding author: Joshua (Sung) H. You, Department of Physical Therapy, Yonsei University, Wonju, Kangwon-do, Korea. Tel.: +82 337602476; Fax: +82 337602496; E-mail: neurorehab@yonsei.ac.kr.
}

0928-7329/17/\$35.00 (c) 2017 - IOS Press and the authors. All rights reserved

This article is published online with Open Access and distributed under the terms of the Creative Commons Attribution NonCommercial License (CC-BY-NC 4.0). 
posterior "pelvic tilting exercise" to selectively activate the deep core muscles [2]. In children with cerebral palsy (CP), core muscle strengthening of weak abdominal muscles has been shown to improve core muscle co-activation, joint stability, associated dynamic weight shifting, and postural and equilibrium upright reactions [3]. NDT-based exercise was found to be effective for increasing proximal stability, which is essential for postural control [4]. However, it is unclear whether NDT-based core exercise has such therapeutic effects in stroke patients. Furthermore, as NDT-based core stabilization exercise emphasizes 'conscious' and active core stabilization to upright the spine, it might not be conducive for stroke patients if they are unable to follow the instructions due to cognitive impairment or reduced somatosensory or movement awareness [3,5]. As a way to overcome this limitation, dynamic neuromuscular stabilization (DNS)-based core stabilization exercise has recently been employed to stimulate subconsciously and reflexively, rather than consciously or cognitively, train deep core muscles such as the diaphragm and the transversus abdominis/internal oblique (TrA/IO). DNS was developed based on neurodevelopmental kinesiology and reflex-mediated core stabilization concepts [6]. DNS utilizes the subconscious stimulation of special zones (chest zones) to reflexively mediate the diaphragm and other core stabilization muscles, which is extremely effective for individuals with reduced somatosensory or movement awareness [7]. However, there is a dearth of objective therapeutic evidence supporting the efficacy of NDT or DNS for core stabilization. Hence, the present study was undertaken to examine the therapeutic effects of NDT and DNS interventions on muscular activity in core muscles and the associated core stability and muscle thickness, assessed with electromyography (EMG), a pressure biofeedback unit (PBU), and ultrasound imaging, respectively. We hypothesized that DNS would result in greater muscular activity and associated core stability than NDT.

\section{Materials and methods}

\subsection{Materials and procedure}

Five healthy adults (mean age $26.1 \pm 1.1$ years, male: 5 ) and five hemiparetic stroke patients (mean age $50.8 \pm 6.8$ years, female: 2 male: 3 ) were recruited. All participants provided informed consents and underwent both NDT and DNS core exercise training and subsequent ultrasound and EMG measurements. Inclusion criteria for stroke patients entailed a first-ever stroke (mean months $2.80 \pm 0.83$ ) and an ability to follow the instructions (Mini-mental State Examination-Korea, MMSE-K = mean score $26.81 \pm 1.53)$. However, stroke patients with severe visual hemianopsia, cognitive impairments, or surgical history were excluded.

A surface wireless EMG (Telemyo 2400 T, Noraxon, Scottsdale, AZ, USA) was used to determine the core muscle activity of the transversus abdominis/internal oblique (TrA/IO), external oblique (EO), and rectus abdominis (RA) muscles. Prior to electrode placement, the surface of the skin was cleaned with alcohol. EMG electrodes (with 1-cm inter-electrode spacing) were placed approximately $3 \mathrm{~cm}$ lateral to the umbilicus to record rectus abdominis activity, approximately $2 \mathrm{~cm}$ horizontally inward and distal to the anterior superior iliac spine (ASIS) to record TrA/IO activity, and laterally halfway between the lower rib cage and the iliac crest to record EO activity on the dominant side [8,9]. EMG data were acquired at a sampling rate of $1500 \mathrm{~Hz}$ and band-pass filtered at 20-450 Hz. The root mean square (RMS) was computed to normalize the EMG data (Fig. 1).

All participants practiced both NDT and DNS core stabilization exercise for 20 minutes per each session over 3 consecutive sessions. Once they were familiarized with the NDT and DNS core stabilization 


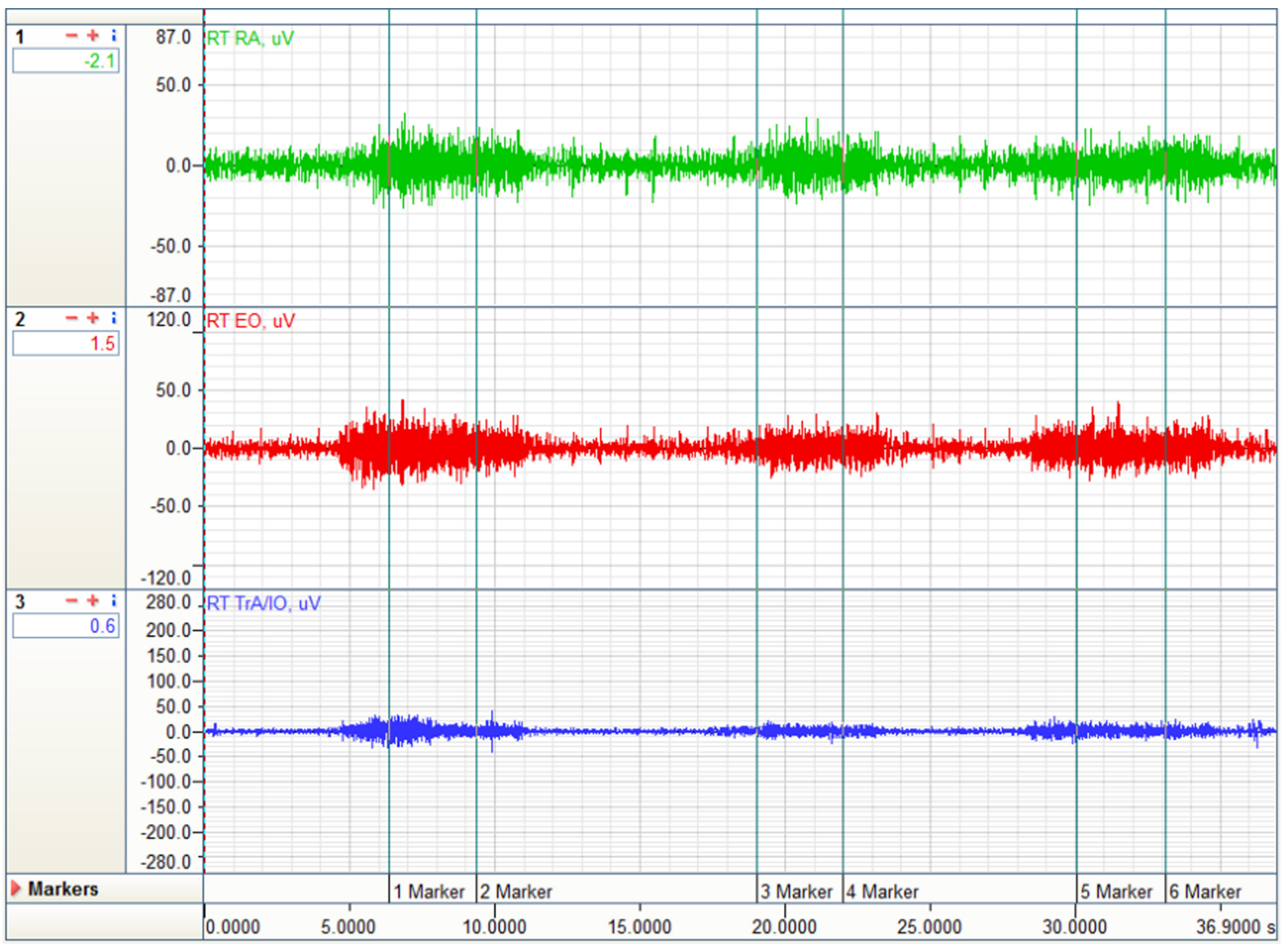

Fig. 1. EMG amplitudes of the TrA/IO, EO, and RA muscles. TrA/IO: transversus abdominis/internal oblique; EO: exter-nal oblique; RA: rectus abdominis.

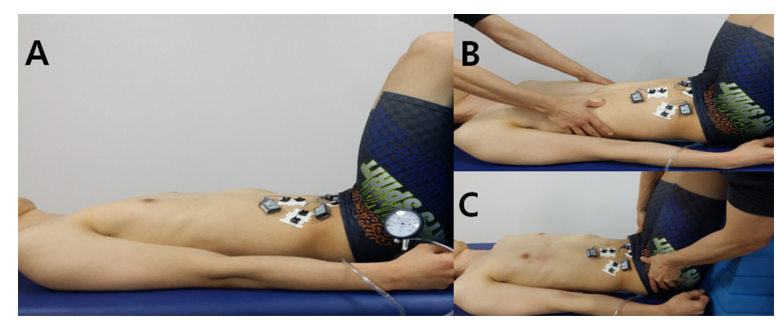

Fig. 2. Exercises were implemented with participants lying supine with $90^{\circ}$ hip and knee flexion, with the PBU under the lumbar spine. Three positions were used - A: resting position (crook lying), B: DNS, C: NDT.

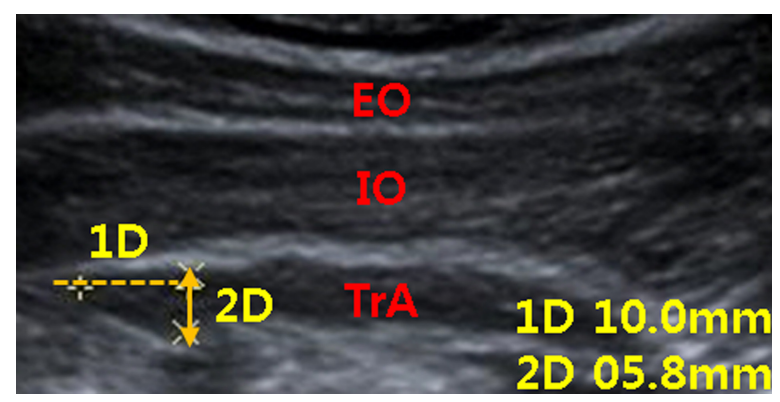

Fig. 3. Ultrasound muscle thickness data of the TrA muscle. TrA: transversus abdominis; IO: internal oblique; EO: external oblique.

exercise, they underwent the EMG and ultrasound imaging tests during the NDT and DNS core stabilization exercise conditions. Each subject was asked to lie supine with $90^{\circ}$ flexion of the hip and knee and was supported with a regular pad (Fig. 2). A PBU (Stabilizer, Chattanooga South Pacific, Australia) was then placed under the lumbar spine and inflated to $40 \mathrm{mmHg}$ to measure core stability [10]. For 
Table 1

EMG amplitude data $(\mu \mathrm{V})$ between NDT and DNS exercises in healthy and stroke subjects $(N=10)$

\begin{tabular}{|c|c|c|c|c|c|c|}
\hline \multirow[t]{2}{*}{ EMG } & \multicolumn{3}{|c|}{ Healthy subjects $(n=5)$ Median (Range) } & \multicolumn{3}{|c|}{ Stroke subjects $(n=5)$ Median (Range) } \\
\hline & NDT & DNS & $\begin{array}{l}\text { Mann-Whitney U } \\
\text { (p-value) }\end{array}$ & NDT & DNS & $\begin{array}{l}\text { Mann-Whitney U } \\
\text { (p-value) }\end{array}$ \\
\hline TrA/IO & $6.23(1.76-8.60)$ & $11.10(6.55-12.20)$ & $0.03^{*}$ & $2.48(1.53-3.34)$ & $3.67(2.71-5.93)$ & $0.03^{*}$ \\
\hline EO & $7.21(4.80-10.80)$ & $3.74(2.74-4.50)$ & $0.008^{*}$ & $3.84(2.61-8.80)$ & $3.72(2.47-4.85)$ & 0.42 \\
\hline RA & $8.73(4.30-14.20)$ & $4.21(2.63-4.70)$ & $0.01^{*}$ & $5.09(3.64-7.61)$ & $4.73(3.04-6.35)$ & 0.42 \\
\hline
\end{tabular}

NDT: neurodevelopmental treatment; DNS: dynamic neuromuscular stabilization; TrA/IO: transversus abdominis/internal oblique; EO: external oblique; RA: rectus abdominis. ${ }^{*}$ Significant at $p<0.05$.

the NDT-based core exercise condition, the subject was positioned in a slightly posterior tilted position, actively pulled his/her belly toward the lumbar spine, and maintained that position. The pressure change reflected on the PBU was recorded and compared with that of the DNS exercise test. For the DNS exercise condition, while the subject was in the same position, his/her chest zone (between the 5th and 6th intercostal spaces, the intersection between the mammillary line and the horizontal xiphoid process) was stimulated with pressure from a therapist's thumb (about $20 \mathrm{mmHg}$ ), with the stimulation vector aimed toward the contralateral ASIS, and the reflex-mediated core stabilization response was measured with the PBU [6].

An ultrasound system (Siemens Acuson S2000, Siemens Healthcare, Erlangen, Germany) was used to measure the thickness of the TrA muscle and thus determine core stability (Fig. 3). A curved linear transducer (Acuson-6C1) with a frequency of $10 \mathrm{MHz}$ was used in B-mode and applied on the anterolateral abdominal wall, lateral to the midline and halfway between the iliac crest and the inferior border of the rib cage. A real-time ultrasound image was displayed on the monitor, and the clearest image was captured at the end of expiration. A horizontal reference line was drawn $1 \mathrm{~cm}$ from the myofascial junction of the TrA muscle to determine the thickness of the TrA. The muscle thickness was measured three consecutive times, and the values were averaged for further statistical analysis [11].

\subsection{Statistical analysis}

Data are reported as median and range and were compared using nonparametric Mann - Whitney U test and Wilcoxon signed rank test. The Wilcoxon signed rank test was used to identify any statistically significant differences in EMG amplitude, muscle thickness, or PBU pressure between the pretest and posttest measurements within each group. The Mann-Whitney U-test was used to identify any statistically significant differences in EMG amplitude, muscle thickness, or PBU pressure between the groups with and without hemiparetic stroke. The level of statistical significance was set at $p<0.05$.

\section{Results}

\subsection{EMG amplitude data}

EMG amplitude data were compared between the NDT and DNS conditions in the healthy subjects and stroke subjects (Table 1). Healthy subjects exhibited greater activation in the deep core $\operatorname{TrA} / \mathrm{IO}(p<$ $0.05)$ but less activation in the superficial EO $(p<0.01)$ and RA $(p<0.05)$ muscles during DNS than during NDT. Stroke subjects displayed greater activation in the deep core TrA/IO muscles during DNS than during NDT $(p<0.05)$. 
Table 2

TrA muscle thickness data $(\mathrm{mm})$ between NDT and DNS exercises in healthy and stroke subjects $(N=10)$

\begin{tabular}{|c|c|c|c|c|c|c|}
\hline \multirow{2}{*}{$\begin{array}{l}\operatorname{Tr} \mathrm{A} \\
\text { thickness }\end{array}$} & \multicolumn{3}{|c|}{ Healthy subjects $(n=5)$ Median (Range) } & \multicolumn{3}{|c|}{ Stroke subjects ( $n=5)$ Median (Range) } \\
\hline & NDT & DNS & $\begin{array}{c}\text { Mann-Whitney U } \\
\text { (p-value) }\end{array}$ & NDT & DNS & $\begin{array}{c}\text { Mann-Whitney U } \\
\text { (p-value) }\end{array}$ \\
\hline Pretest & $1.60(0.85-3.40)$ & $1.60(0.85-3.40)$ & 1.00 & $1.90(0.80-3.40)$ & $1.90(0.80-3.40)$ & 1.00 \\
\hline Posttest & $2.41(1.46-3.70)$ & $2.59(1.68-4.00)$ & 0.69 & $2.80(1.10-4.80)$ & $2.40(2.00-4.00)$ & 0.84 \\
\hline $\begin{array}{l}\text { Wilcoxon } \\
\text { (p-value) }\end{array}$ & $0.04^{*}$ & $0.04^{*}$ & & $0.04^{*}$ & $0.04^{*}$ & \\
\hline
\end{tabular}

NDT: neurodevelopmental treatment; DNS: dynamic neuromuscular stabilization; TrA: transversus abdominis. ${ }^{*}$ Significant at $p<0.05$.

Table 3

PBU pressure change data $(\mathrm{mmHg})$ between NDT and DNS exercises in healthy and stroke subjects $(N=10)$

\begin{tabular}{|c|c|c|c|c|c|c|}
\hline \multirow{2}{*}{$\begin{array}{l}\mathrm{PBU} \\
\text { pressure }\end{array}$} & \multicolumn{3}{|c|}{ Healthy subjects $(n=5)$ Median (Range) } & \multicolumn{3}{|c|}{ Stroke subjects $(n=5)$ Median (Range) } \\
\hline & NDT & DNS & $\begin{array}{c}\text { Mann-Whitney U } \\
\text { (p-value) }\end{array}$ & NDT & DNS & $\begin{array}{c}\text { Mann-Whitney U } \\
\text { (p-value) }\end{array}$ \\
\hline Pretest & 40 & 40 & 1.00 & 40 & 40 & 1.00 \\
\hline Posttest & $60(50-68)$ & $64(52-80)$ & 0.42 & $58(50-86)$ & $60(54-84)$ & 0.54 \\
\hline $\begin{array}{l}\text { Wilcoxon } \\
\text { (p-value) }\end{array}$ & $0.04^{*}$ & $0.04^{*}$ & & $0.04^{*}$ & $0.04^{*}$ & \\
\hline
\end{tabular}

NDT: neurodevelopmental treatment; DNS: dynamic neuromuscular stabilization. ${ }^{*}$ Significant at $p<0.05$.

\subsection{Intervention-related change in TrA thickness}

TrA muscle thickness data were compared between the pretest and posttest conditions in the healthy subjects and stroke subjects (Table 2). TrA muscle thickness was significantly greater in the posttest than in the pretest under both conditions in both groups $(p<0.05)$. However, no significant difference in $\operatorname{Tr} A$ thickness was observed between the groups at the posttest.

\subsection{Core stability change}

The median core stability values were compared between the pretest and posttest conditions in the healthy subjects and stroke subjects (Table 3 ). PBU core stability was significantly greater posttest than pretest under both conditions in both groups $(p<0.05)$. However, no significant difference in pressure data was observed between the groups at the posttest.

\section{Discussion}

The purpose of the present investigation was to compare the therapeutic effects of NDT and DNS exercises on EMG activation patterns, TrA muscle thickness values, and associated changes in core stability in healthy subjects and stroke patients. As anticipated, EMG analysis demonstrated that hemiparetic stroke subjects exhibited greater activation of the deep core TrA/IO muscles during DNS than during NDT, while healthy subjects displayed greater activation in the deep core TrA/IO but less activation in the superficial EO and RA muscles during DNS than during NDT. Thus, whether or not they had experienced hemiparetic stroke, subjects demonstrated better TrA/IO muscle activity during DNS than during NDT. In the healthy group, DNS exercise increased core stabilization by synchronously activating the TrA/IO while inhibiting the EO and RA muscles relative to conventional NDT exercise. This 
finding suggests that DNS might have stimulated the underactive deep core TrA/IO muscles in hemiparetic patients, while reciprocally reducing the overactive superficial EO and RA muscles in healthy subjects. In fact, the relative changes in EMG amplitude were far greater during DNS than during NDT, suggesting that this change in deep core muscle activation was facilitated by reflex-mediated stimulation of the chest zones during DNS [7]. In stroke patients, this reflex-mediated stimulation might have augmented their impaired sense of proprioceptive movement, which is responsible for the co-activation of core muscles [12]. These results support the theoretical assumption that selective stimulation of the chest zones during DNS can evoke core stabilization, which is mediated by the monosynaptic reflexive activation system [6]. However, this reflex-mediated stabilization was not apparent in stroke patients during conventional NDT, which utilizes cortical and conscious awareness of proprioceptive kinesthetic inputs during the realignment of abnormal pelvic girdle movement.

Ultrasound imaging and PBU data demonstrated that TrA muscle thickness and core stability were significantly greater posttest than pretest under both conditions in both groups. However, no significant differences in TrA thickness or core stability were observed between the groups at the posttest. These findings suggest that the improvements in TrA muscle activity were associated with increased muscle thickness and core stability, further corroborating the DNS and NDT treatment concepts. DNS, which is based on neurodevelopmental kinesiology, emphasizes core stabilization through the co-activation of the diaphragm-TrA/IO-pelvic floor-multifidus muscles in coordination with the superficial core muscles, which generates sufficient intra-abdominal pressure (IAP) to dynamically stabilize the spine [13,14]. As the participant inhales, the diaphragm descends and reflexively stimulates the other deep core chain muscles (the TrA/IO-pelvic floor-multifidus) eccentrically. The eccentrically-activated muscles concentrically activate and produce IAP, resulting in the core stabilization and associated postural stability required during dynamic movements $[7,15]$. NDT focuses on control of the pelvic girdle and facilitates core stabilization by realigning the abnormal movement and positioning of the pelvic girdle. This normalization of the proprioceptive inputs in the lumbopelvic region facilitates the selective concentric activation of the deep TrA/IO core muscles. The selective concentric contraction of the local abdominal muscles appears to increase core stabilization, thereby generating sufficient core stabilization $[16,17]$; indeed, this was reflected in our ultrasound imaging, EMG, and PBU measurements. Such phenomena have also been well-reflected in recent studies, which demonstrated the effects of core stability strength exercises on muscle activity, static and dynamic balance, and coordination in healthy subjects and stroke patients $[18,19]$. Although the present preliminary study provided novel, promising evidence regarding the effects of NDT-based and DNS-based core stabilization exercises on postural core stabilization, it might be difficult to generalize our findings due to the sample size. Future research should be conducted with a larger sample size in order to increase the experimental robustness and generalizability of our findings. Healthy subjects were used as normal case controls since this study is a case condtrol study desgin. However, the age and gender matched controls would be more desirable. Nevertheless, our present data demonstrated that our DNS and ADIM treatments performed on healthy adults improved TrA thickness, it may be an useful intervention for children and adolescents with or without scoliosis because they have similar TrA muscle as in adults [20,21].

\section{Conclusion}

This is the first clinical study to compare the effects of NDT and DNS core stabilization exercises on core muscle activity, thickness, and associated core stability between groups with and without hemiparetic stroke. We demonstrated that DNS was superior to NDT in its effect on TrA/IO activity. However, both DNS and NDT were beneficial for improving TrA/IO muscle thickness and core stability. 
Clinically, this study provides important insights for clinicians who wish to design effective therapeutic core exercise for individuals who display impaired postural core stability, with and without hemiparetic stroke.

\section{Acknowledgments}

This study was supported by an intramural grant from Chungnam National University Hospital, Daejeon, Republic of South Korea, and by an extramural Brain Korea 21 PLUS Project Grant (2016-510009) from the Korean Research Foundation.

\section{Conflict of interest}

None to report.

\section{References}

[1] Granacher U, Lacroix A, Muehlbauer T, Roettger K, Gollhofer A. Effects of core instability strength training on trunk muscle strength, spinal mobility, dynamic balance and functional mobility in older adults. Gerontology. 2013; 59(2): 105-13.

[2] Gjelsvik BEB. The Bobath concept in adult neurology: Thieme; 2008: 103-4, 111-2.

[3] Sterba JA, Rogers BT, France AP, Vokes DA. Horseback riding in children with cerebral palsy: effect on gross motor function. Developmental Medicine and Child Neurology. 2002; 44(5): 301-8.

[4] Hafsteinsdottir TB, Algra A, Kappelle LJ, Grypdonck MH, Dutch NDTSG. Neurodevelopmental treatment after stroke: a comparative study. Journal of Neurology, Neurosurgery, and Psychiatry. 2005; 76(6): 788-92.

[5] Gontkovsky ST, McDonald NB, Clark PG, Ruwe WD. Current directions in computer-assisted cognitive rehabilitation. NeuroRehabilitation. 2002; 17(3): 195-9.

[6] Kolar P. Clinical rehabilitation: Alena Kobesová; 2014: 252-8.

[7] Frank C, Kobesova A, Kolar P. Dynamic neuromuscular stabilization and sports rehabilitation. International Journal of Sports Physical Therapy. 2013; 8(1): 62-73

[8] Chon SC, Chang KY, You JS. Effect of the abdominal draw-in maneuver in combination with ankle dorsiflexion in strengthening the transverse abdominal muscle in healthy young adults: a preliminary, randomized, controlled study. Physiotherapy. 2010; 96(2): 130-6.

[9] Escamilla RF, Lewis C, Bell D, Bramblet G, Daffron J, Lambert S, et al. Core muscle activation during Swiss ball and traditional abdominal exercises. The Journal of Orthopaedic and Sports Physical Therapy. 2010; 40(5): 265-76.

[10] Drysdale CL, Earl JE, Hertel J. Surface electromyographic activity of the abdominal muscles during pelvic-tilt and abdominal-hollowing exercises. Journal of Athletic Training. 2004; 39(1): 32-6.

[11] Whittaker JL. Ultrasound imaging of the lateral abdominal wall muscles in individuals with lumbopelvic pain and signs of concurrent hypocapnia. Manual Therapy. 2008; 13(5): 404-10.

[12] Thikey H, Grealy M, van Wijck F, Barber M, Rowe P. Augmented visual feedback of movement performance to enhance walking recovery after stroke: study protocol for a pilot randomised controlled trial. Trials. 2012; 13: 163.

[13] Noh DK, Lee JJ, You JH. Diaphragm breathing movement measurement using ultrasound and radiographic imaging: a concurrent validity. Bio-medical Materials and Engineering. 2014; 24(1): 947-52.

[14] Kolar P, Neuwirth J, Sanda J, Suchanek V, Svata Z, Volejnik J, et al. Analysis of diaphragm movement during tidal breathing and during its activation while breath holding using MRI synchronized with spirometry. Physiological Research. 2009; 58(3): 383-92.

[15] Chaitow L, Gilbert C, Morrison D. Recognizing and treating breathing disorders: Elsevier Health Sciences; 2014: 11-22.

[16] Vezina MJ, Hubley-Kozey CL. Muscle activation in therapeutic exercises to improve trunk stability. Archives of Physical Medicine and Rehabilitation. 2000; 81(10): 1370-9.

[17] Wang RY, Chen HI, Chen CY, Yang YR. Efficacy of Bobath versus orthopaedic approach on impairment and function at different motor recovery stages after stroke: a randomized controlled study. Clinical Rehabilitation. 2005; 19(2): 155-64. 
S106 H.S. Yoon and J.H. You / Reflex-mediated DNS in stroke patients: EMG processing and ultrasound imaging

[18] Yu SH, Park SD. The effects of core stability strength exercise on muscle activity and trunk impairment scale in stroke patients. Journal of Exercise Rehabilitation. 2013; 9(3): 362-7.

[19] Chung EJ, Kim JH, Lee BH. The effects of core stabilization exercise on dynamic balance and gait function in stroke patients. Journal of Physical Therapy Science. 2013 Jul; 25(7): 803-806.

[20] Linek P, Saulicz E, Wolny T, Myśliwiec A, Kokosz M. Lateral abdominal muscle size at rest and during abdominal drawing-in manoeuvre in healthy adolescents. Manual Therapy. 2015; 20(1): 117-23.

[21] Linek P, Saulicz E, Kuszewski M, Wolny T. Ultrasound assessment of the abdominal muscles at rest and during the ASLR test among adolescents with scoliosis. Journal of Spinal Disorders and Techniques. 2014. 